\title{
Inhibition of autophagy potentiates the proliferation inhibition activity of microRNA-7 in human hepatocellular carcinoma cells
}

\author{
YANNA WANG $^{1 *}$, QIAOLING WANG ${ }^{1 *}$ and JIQING SONG ${ }^{2}$ \\ ${ }^{1}$ Department of Infectious Diseases, Yantai Hospital For Infectious Diseases, Yantai, Shandong 264001; \\ ${ }^{2}$ Nursing Department of Yantai Yeda Hospital, Yantai, Shandong 264006, P.R. China
}

Received October 19, 2015; Accepted April 21, 2017

DOI: $10.3892 / \mathrm{ol} .2017 .6573$

\begin{abstract}
MicroRNAs (miRNAs/miRs) are important molecules that are able to regulate multiple cellular processes in cancer cells. miR-7 has been previously identified as a tumor suppressive miRNA in several types of cancer. The aim of the present study was to investigate whether miR-7 is able to regulate autophagy in hepatocellular carcinoma (HCC) cells. It was identified that miR-7 was significantly downregulated in tumor tissues compared with adjacent normal tissues. Overexpression of miR-7 inhibited cell proliferative activity, which was partially reversed by miR-7 inhibitor. In addition, overexpression of miR-7 significantly induced an increasen in autophagic activity, and luciferase activity assay and western blot analysis identified that mammalian target of rapamycin (mTOR) was a direct target of miR-7. In addition, inhibition of autophagy by 3-methyladenine resulted in a marked enhancement of the proliferation inhibition effect of miR-7. In conclusion, miR-7 was identified to induce proliferation inhibition and autophagy in HCC cells by targeting mTOR, and inhibition of autophagy may be utilized to enhance the antitumor activity of miR-7.
\end{abstract}

\section{Introduction}

Hepatocellular carcinoma (HCC) is one of the leading causes of cancer-associated mortality worldwide (1). HCC is often detected at an advanced stage and exhibits poor prognosis (2). The pathogenesis of HCC is incompletely understood, and an effective treatment for $\mathrm{HCC}$ remains

Correspondence to: Mr. Jiqing Song, Nursing Department of Yantai Yeda Hospital, 11 Taishan Road, Yantai, Shandong 264006, P.R. China

E-mail: songjiqing789@126.com

*Contributed equally

Key words: hepatocellular carcinoma, microRNA-7, autophagy, mammalian target of rapamycin a requirement. As such, it is of great importance to elucidate the molecular mechanisms underlying the biological behavior of HCC cells.

Autophagy is a catabolic process that is induced under various conditions of stress (3). Despite its diverse roles proposed by several studies, evidence suggests that autophagy is critical for cell survival (4), and aberrant autophagic activity is implicated in the development, progression and drug resistance of HCC (5-7). Mammalian target of rapamycin (mTOR) tethers several upstream signals to exert an inhibitory effect on autophagy (8). Inhibition of mTOR signaling is one of the potential strategies for HCC therapy $(9,10)$; however, the role of autophagy in this context is not fully understood.

MicroRNAs (miRNAs/miRs) have emerged as a class of key regulators in multiple types of cancers $(11,12)$. mRNAs canonically match with the 3'untranslated region (UTR) of targeted mRNAs, which consequently results in gene silencing (13). A broad spectrum of miRNAs have been implicated in the diagnostic and therapeutic strategies for HCC (14). It has been reported that miR-7 is downregulated in various types of cancer and functions as a tumor suppressor $(15,16)$. Previous studies have identified a number of oncogenes to be targeted by miR-7 (17-20). In HCC, it exhibits anticancer activity by causing cell cycle arrest via targeting critical genes in cell cycle progression (17). However, whether miR-7 regulates other important cell machinery, such as autophagy, remains unknown. In particular, miR-7 contributes to the inhibition of tumor growth and metastasis by modulating the phosphoinositide 3-kinase/protein kinase B (Akt)/mTOR signaling pathway (21), a major signaling pathway involved in the regulation of autophagy induction, indicating its potential involvement in autophagy regulation.

In the present study it was identified that miR-7 is significantly downregulated in HCC tissues compared with the normal adjacent tissue. Supplementation of miR-7 in HepG2 cancer cells inhibited the proliferation of the cells. In addition to the proliferation inhibition effect of miR-7, autophagy was identified to be induced by miR-7 at the same time, and inhibition of mTOR was responsible for the autophagy induction. Most notably, suppression of autophagy enhanced the proliferation inhibition effect of miR-7. Therefore, the results of the present study revealed a novel mechanism by which miR-7 
regulates autophagy, and modulation of autophagy may be a feasible approach to enhance the antitumor activity of miR-7.

\section{Materials and methods}

Clinical samples. A total of 17 HCC tissues (age, 32-65; 9 males and 8 females) and paired adjacent tissues were obtained under the supervision of the ethics committee of the local hospital (Yantai City Hospital for Infectious Diseases, Shandong, China) between March 2015 and June 2015. All the patients did not receive any chemotherapy prior to surgery. The diagnoses of HCC were histologically confirmed by three independent pathologists, and the negative resection margin was confirmed during the surgery. Tissues were snap-frozen in liquid nitrogen. All patients provided written informed consent for the study.

Cell culture and 3-methyladenine (3-MA) treatment. The human HCC HepG2 and Huh-7 cell lines and human embryonic kidney HEK293-T cell line used in the present study were obtained from the American Type Culture Collection (Manassas, VA, USA). The cells were maintained in Dulbecco's modified Eagle's medium (DMEM; Hyclone; GE Healthcare, Logan, UT, USA) with $10 \%$ fetal bovine serum (FBS; Hyclone; GE Healthcare). Cells were cultured at $37^{\circ} \mathrm{C}$ in a humidified atmosphere containing $5 \% \mathrm{CO}_{2}$, and the medium was changed every 3 days. 3-MA was purchased from Sigma-Aldrich (Merck KGaA, Darmstadt, Germany) and dissolved in the culture medium, and a $5 \mathrm{mM}$ final concentration was applied to cells to inhibit autophagy. For autophagy flux assay, co-treatment with $20 \mu \mathrm{M}$ chloroquine (C6628; Sigma-Aldrich; Merck KGaA) was applied.

Transfection. The miRNA mimic for miR-7 (miR10004553-1-5) and its exact antisense inhibitor (miR200004553-1-5) were obtained from Guangzhou RiboBio Co.,Ltd. (Guangzhou, China). The nucleotides were transfected using Lipofectamine 2000 (Invitrogen; Thermo Fisher Scientific, Inc., Waltham, MA, USA) at a final concentration of $50 \mathrm{nM}$, according to the manufacturer's protocol. miRNAs were diluted in DMEM and incubated with a proper amount ( $10 \mu \mathrm{l}$ for one 6 -well plate) of Lipofectamine 2000 at $37^{\circ} \mathrm{C}$ for $20 \mathrm{~min}$. The Lipofectamine 2000 -miRNA mixture was then added to each well of a 6 -well plate; after $12 \mathrm{~h}$ of incubation in serum-free conditions, the medium was refreshed with complete medium containing 10\% FBS.

Cell proliferation assay. To determine the cell proliferation, a Cell Counting Kit-8 (CCK-8; Wuhan Boster Biological Technology, Ltd., Wuhan, China) was used. The experiment was conducted according to the manufacturer's protocol. The absorbance value at $450 \mathrm{~nm}$ was determined using a plate reader as a measure of proliferative activity.

Reverse transcription-polymerase chain reaction (RT-PCR). Following treatment, cells were collected and lysed with $1 \mathrm{ml}$ TRIzol reagent (Invitrogen; Thermo Fisher Scientific, Inc.). Chloroform $(0.2 \mathrm{ml})$ was then added to the tube and mixed vigorously with TRIzol reagent. RNA was precipitated using isopropyl alcohol from the upper phase and washed using $75 \%$ ethanol. The RNA samples were resolved in nuclease-free double-distilled water (Promega Corporation, Madison, WI,

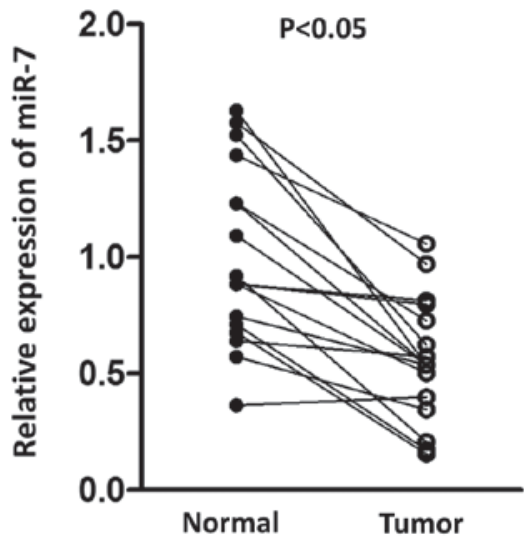

Figure 1. Differential expression of miR-7 in hepatocellular carcinoma tissues and paired adjacent normal tissues. A significant decrease in miR-7 expression was observed in tumor tissues compared with normal tissues $(\mathrm{P}<0.05) . \mathrm{n}=17 . \mathrm{miR}-7$, microRNA-7.

USA). miR-7 was then reverse-transcribed using a specific stem-loop primer, followed by a SYBR Green-based amplification protocol. Reverse transcription was performed with the GoScript reverse transcription kit (Promega Corporation), at $25^{\circ} \mathrm{C}$ for $5 \mathrm{~min}$, and then $42^{\circ} \mathrm{C}$ for $1 \mathrm{~h}$. PCR was performed using the GoTaq aPCR Master Mix (Promega Corporation), which included the DNA polymerase. The thermocycling conditions were as follows: $95^{\circ} \mathrm{C}$ for $20 \mathrm{sec}, 58^{\circ} \mathrm{C}$ for $20 \mathrm{sec}$ and $72^{\circ} \mathrm{C}$ for $20 \mathrm{sec}$, for 40 cycles. Small nuclear RNA U6 was used as an internal control. The primer sets for miR-7 and U6 were all obtained from Guangzhou RiboBio Co., Ltd. (Guangzhou, China; cat. no., miRQ0000252-1-2). All experiments were repeated three times. Quantification was performed using the $2^{-\Delta \Delta \mathrm{Cq}}$ method (22).

Western blot analysis. Total protein from the treated cells was obtained by homogenizing cells with radioimmunoprecipitation assay buffer (Thermo Fisher Scientific, Inc.). The protein concentration was quantified by a bicinchoninic acid kit (Thermo Fisher Scientific, Inc.). Protein $(\sim 40 \mu \mathrm{g})$ was loaded on $15 \%$ SDS-PAGE gels. Following electrophoresis, the protein was blotted onto a polyvinylidene fluoride membrane. The membrane was blocked with non-fat milk at $37^{\circ} \mathrm{C}$ for $1 \mathrm{~h}$, and incubated with primary antibodies [LC3 (cat. no., 3868; dilution, 1:1,000), mTOR (cat. no., 2983; dilution, 1:1,000), p62 (cat. no., 8025; dilution, 1:1,000), p-mTOR (cat. no., 5536; dilution, 1:1,000) and $\beta$-actin (cat. no., TA310155; dilution, 1:2,000)] at $4^{\circ} \mathrm{C}$ for $16 \mathrm{~h}$. Following 5 washes of 5 min with PBS containing $0.5 \%$ Tween-20, membranes were incubated with horseradish peroxidase (HRP)-conjugated secondary antibodies (goat anti-rabbit HRP (Santa Cruz Biotechnology, Inc., Dallas, TX, USA; cat. no., sc-2005; dilution, 1:5,000) and goat anti-mouse HRP (Santa Cruz Biotechnology, Inc.; cat. no., sc-2004; dilution, 1:5,000) at room temperature for $1 \mathrm{~h}$, followed by visualization using an enhanced chemiluminescence detection kit (GE Healthcare Life Sciences, Little Chalfont, UK), according to the manufacturer's protocol. Antibodies against 1A/1B-light chain 3 (LC3), mTOR, phosphorylated mTOR (p-mTOR) and p62 were purchased from Cell Signaling 

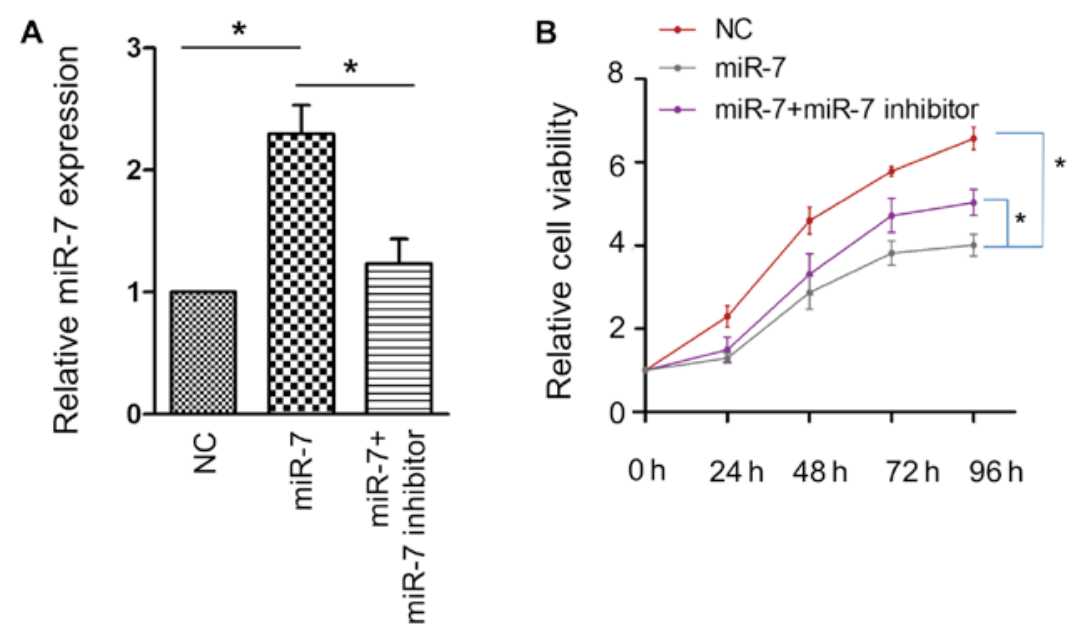

Figure 2. miR-7 inhibits the proliferative activity of HepG2 cells. (A) Quantification of miR-7 expression following transfection with miR-7 or miR-7 + miR-7 inhibitor. (B) Effect of miR-7 and miR-7 + miR-7 inhibitor on cell viability. ${ }^{*} \mathrm{P}<0.05 ; \mathrm{n}=5$ in each group. miR-7, microRNA-7; NC, negative control.

Technology, Inc. (Danvers, MA, USA). Antibody against $\beta$-actin was purchased from OriGene Technologies, Inc. (Beijing, China).

Luciferase activity assay. A $500 \mathrm{bp}$ fragment containing the putative binding site of miR-7 was amplified from the cDNA of mTOR, the fragment of mTOR 3'UTR was then inserted into the 3'UTR of PGL-3 firefly reporter (Promega Corporation). HEK293-T cells grown in 24-well plates were transfected with promoter Renilla luciferase-thymidine kinase (internal control), PGL3-mTOR 3'UTR and miR-7 or miR-7 + miR-7 inhibitor, using Lipofectamine 2000 (Thermo Fisher Scientific, Inc.). The luciferase activity was detected after $36 \mathrm{~h}$ using the substrates provided in the Dual Luciferase Reporter Assay kit (Promega Corporation), according to the manufacturer's protocol. The firefly luciferase activity was normalized to Renilla luciferase activity, and the relative luciferase activity in each group was obtained by normalizing the value of (firefly luciferase activity)/(Renilla luciferase activity) to that of the negative control (NC)-transfected group.

Immunofluorescent staining. Cells $\left(2 \times 10^{4} / \mathrm{ml}\right)$ were seeded onto coverslips and cultured in 24 -well plates. Immediately after transfection, cells were incubated at $37^{\circ} \mathrm{C}$ for $48 \mathrm{~h}$. The cells on coverslips were then fixed with PBS containing 4\% paraformaldehyde, and quickly penetrated with $0.3 \%$ Triton $\mathrm{X}-100$ on ice for $5 \mathrm{~min}$. The coverslips were blocked with 5\% bovine serum albumin (Sigma-Aldrich; Merck KGaA) for $1 \mathrm{~h}$ at room temperature. Primary antibody against LC3 (Cell Signaling Technology, Inc.; cat. no., 3868; dilution, 1:200) was then added to the coverslips. Cells were incubated with primary antibody in a humid chamber overnight at $4^{\circ} \mathrm{C}$, and the coverslips were then washed with PBS containing Triton X100 (0.5\%). Alexa Fluor ${ }^{\circledR}$ 594-conjugated anti-rabbit secondary antibody (cat. no., R37117; Thermo Fisher Scientific, Inc.) was added and incubated at room temperature for $1 \mathrm{~h}$ to label the primary antibody for LC3. Cells were counterstained with DAPI and observed by fluorescence microscopy in five fields of view (Nikon Corporation, Tokyo, Japan; magnification, x400).
Statistical analysis. Data are expressed as the mean \pm standard deviation. A paired Student's t-test was used to determine the differential expression of miR-7 in tumor tissues and normal tissues. One-way analysis of variance followed by Dunnett's test was used for multiple comparisons. $\mathrm{P}<0.05$ (two-tailed) was considered to indicate a statistically significant difference. Statistical analysis was performed using SPSS version 19 software (IBM SPSS, Armonk, NY, USA).

\section{Results}

miR-7 expression is downregulated in HCC. Previous studies have demonstrated that miR-7 functions as a tumor suppressor and is downregulated in various types of cancer, including lung cancer and gastric cancer $(19,20,23)$. The present study compared the expression of miR-7 in tumor samples and paired normal tissue. As presented in Fig. 1, a significant decrease in miR-7 was observed in tumor tissue compared with normal tissue, suggesting the potential antitumor role for miR-7 in HCC.

miR-7 inhibits the proliferation of HCC cells. To ascertain the role of miR-7 in HCC, a CCK-8 assay was employed to examine whether miR-7 affects the proliferative activity of the HCC HepG2 cell line. The efficacy of transfection was confirmed using RT-PCR (Fig. 2A). A significant attenuation of cell proliferative activity was observed when miR-7 was overexpressed, and this effect was partially reversed by miR-7 inhibitor (Fig. 2B). These results confirmed that miR-7 serves an antitumor role in HCC.

Overexpression of miR-7 enhances autophagy. Next, the autophagic activity was examined by western blot analysis of the autophagy markers LC3 and p62. Overexpression of miR-7 resulted in increased expression of LC3-phosphatidylethanolamine conjugate (LC3-II), and, accordingly, p62, which is a specific substrate of autophagy (24), was downregulated (Fig. 3A and B). Co-administration of chloroquine (CQ) was used to conduct the autophagy flux assay. Similar to the aforementioned results, the LC3-II level was also increased in the 
A

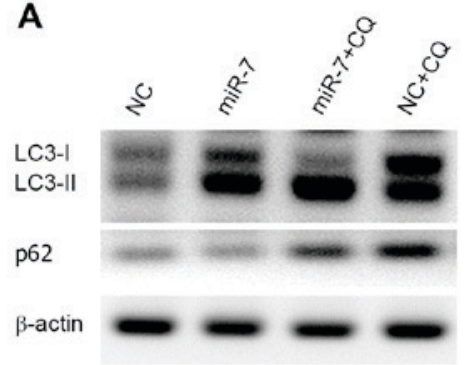

B

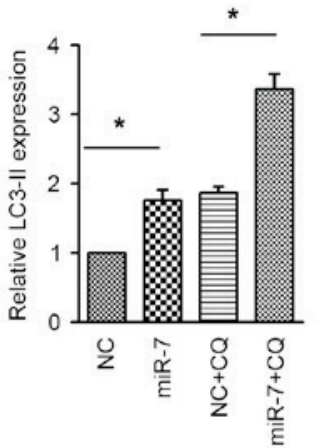

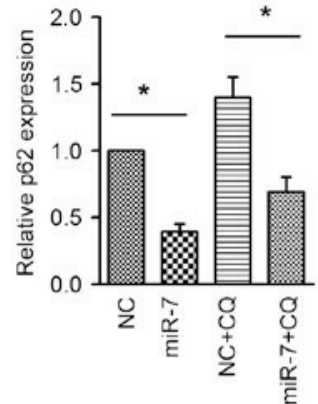

C

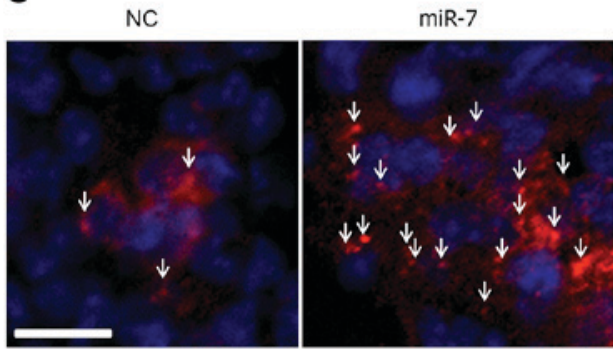

Figure 3. Overexpression of miR-7 induces autophagy in HepG2 cells. (A) Effect of miR-7 on autophagy marker LC3 and p62 with or without treatment with CQ. (B) Quantification of the band density of LC3-II and p62. (C) The autophagosome as demonstrated by LC3 immunofluorescent staining. Arrows indicate the autophagosome dots. Scale bar, $25 \mu \mathrm{m}$. "P<0.05; n=5 in each group. LC3, 1A/1B-light chain 3; LC3-II, LC3-phosphatidylethanolamine conjugate; miR-7, microRNA-7; CQ, chloroquine; $\mathrm{NC}$, negative control.

A

$$
\begin{aligned}
& \text { 3' uguUGUUUUAGUGAUCAGAAGGu 5' hsa-miR-7 } \\
& \text { I II I I I I I I I I I | | } \\
& \text { 5' aguACAAAAGGUCU--GUCUUCCa 3' mTOR (545-566 3'UTR) }
\end{aligned}
$$

C

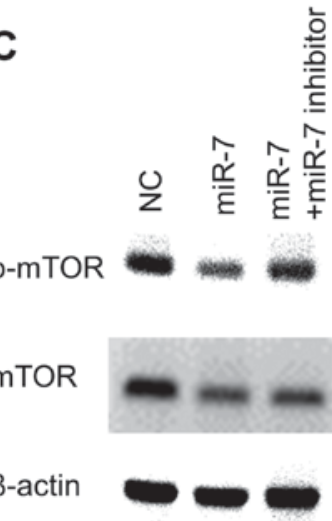

D
B
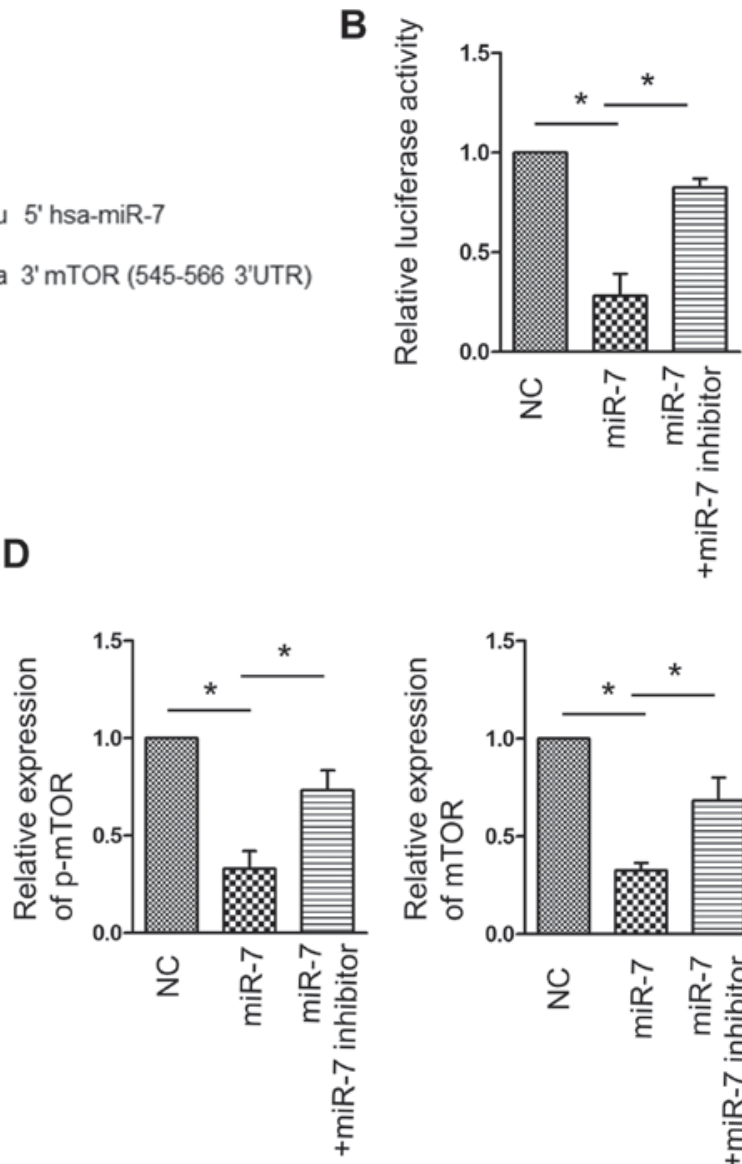

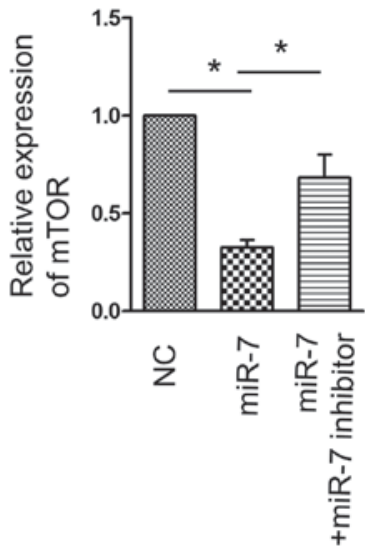

Figure 4. Identification of mTOR as a target of miR-7. (A) Schematic diagram of the base pair match between miR-7 and mTOR 3'UTR. (B) Effect of miR-7 and miR-7 inhibitor on luciferase activity. "P<0.05, $n=5$ in each group. (C) Effect of miR-7 and miR-7 inhibitor on the expression of p-mTOR and mTOR. $\beta$-actin served as an internal control. (D) Statistical analysis of the band density of mTOR and p-mTOR. "P<0.05; $n=5$ in each group. miR-7, microRNA-7; mTOR, mammalian target of rapamycin; p-mTOR, phosphorylated mTOR; NC, negative control; UTR, untranslated region. 

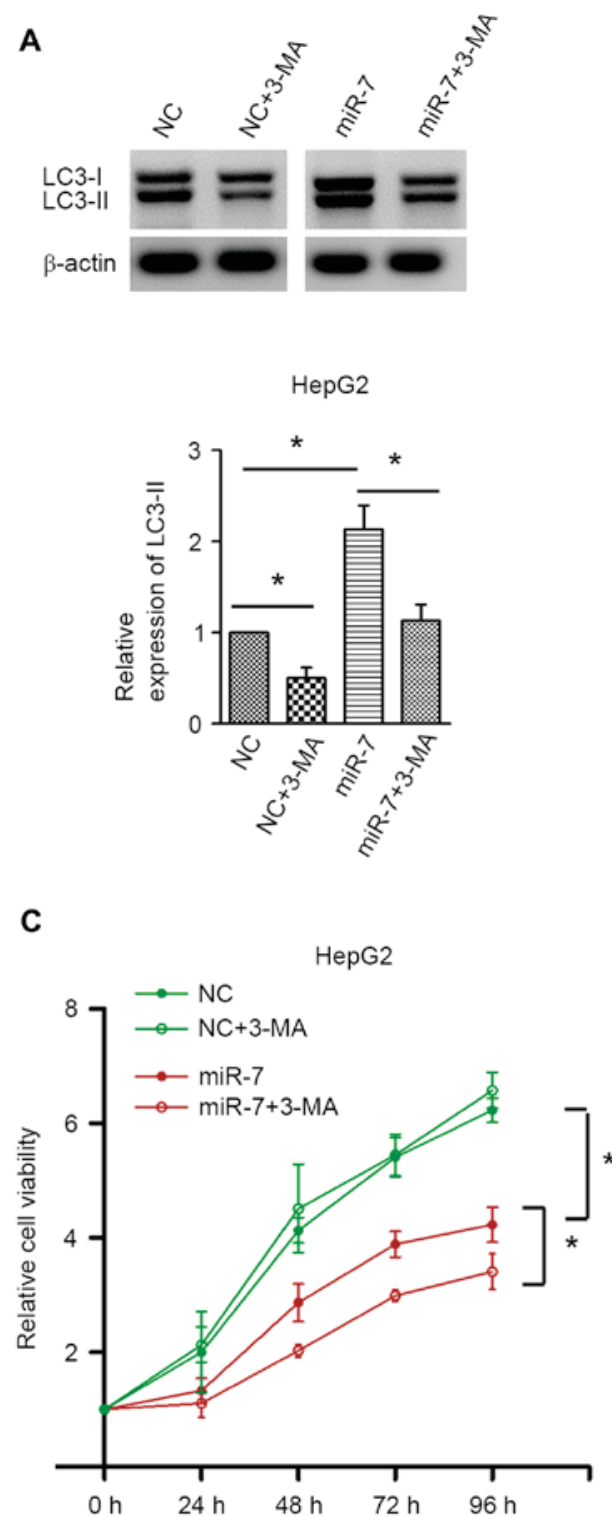
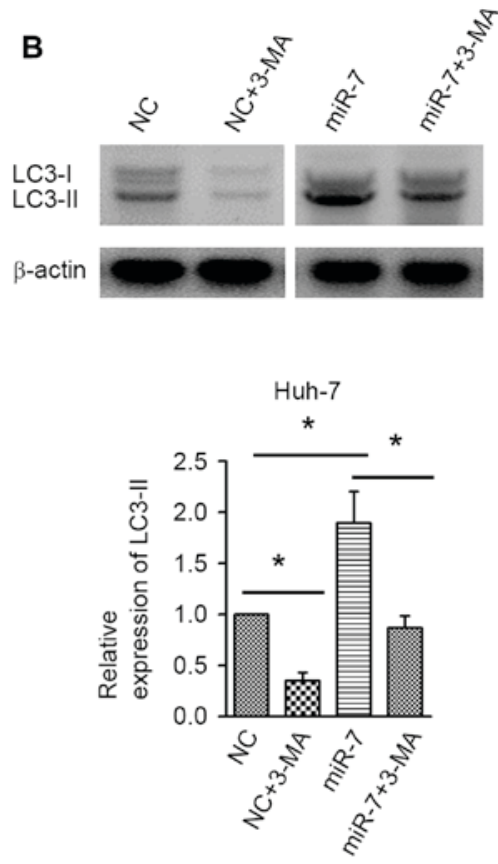

Huh-7

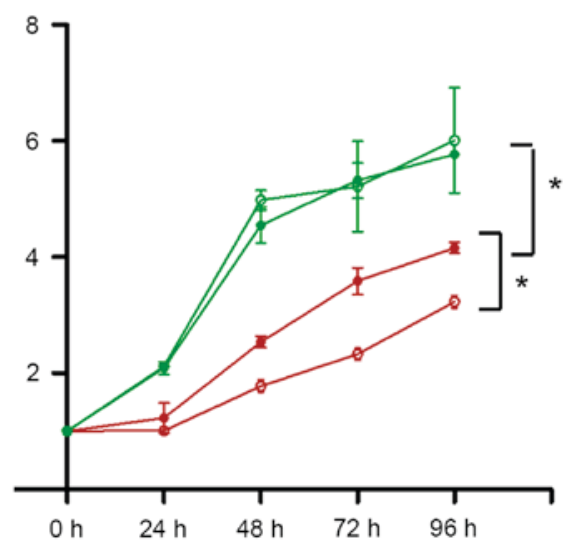

Figure 5. Inhibition of autophagy enhances proliferation inhibition activity of miR-7. Western blot analysis confirmed the induction of autophagy by miR-7 and the inhibition of autophagy by 3-MA in (A) HepG2 and (B) Huh-7 cells, with quantification presented in the histograms. (C) Effect of 3-MA on miR-7-induced proliferation inhibition of HepG2 and Huh-7 cells. "P $<0.05$; $=5$ in each group. miR-7, microRNA-7; 3-MA, 3-methyladenine; NC, negative control; LC3, 1A/1B-light chain 3; LC3-II, LC3-phosphatidylethanolamine conjugate.

miR-7 group in the presence of $\mathrm{CQ}$, indicating the increased autophagy flux (Fig. 3A and B). Immunofluorescent staining of LC3 was performed to visualize the autophagosome, and it was revealed that the autophagosome was aggregated in miR-7-transfected cells (Fig. 3C). These results suggested that overexpression enhances autophagic activity in HCC cells.

mTOR is a target of miR-7. As mTOR is a canonical negative regulator of autophagy, it was hypothesized that miR-7-associated autophagy induction was associated with this pathway. A region in the 3'UTR of the mRNA of mTOR was identified to comprise the seed sequence matching miR-7 (Fig. 4A). A luciferase reporter assay demonstrated that miR-7 overexpression inhibited the luciferase activity of the reporter carrying mTOR 3'UTR, whereas co-transfection of miR-7 and miR-7 inhibitor significantly reversed this effect (Fig. 4B).
Western blot analysis confirmed the inhibition of mTOR expression by miR-7, and consequently, p-mTOR, which represents the kinase activity of mTOR, was also inhibited by miR-7 (Fig. 4C and D). These data indicated that mTOR is a target of miR-7, and inhibition of $\mathrm{mTOR}$ may contribute to miR-7-activated autophagy.

Inhibition of autophagy enhances the proliferation inhibition activity of $m i R-7$. To investigate the exact role of autophagy in HCC, autophagy was inhibited by 3-MA. Western blot analysis confirmed the autophagic inhibition by 3-MA in HepG2 and Huh-7 cells (Fig. 5A and B). 3-MA treatment did not result in significant alterations in cell proliferation in the NC-transfected group. By contrast, inhibition of autophagy by 3-MA significantly decreased the cell proliferative activity upon miR-7 overexpression (Fig. 5C). These data indicated that autophagy induced by miR-7 counteracts the proliferation 
inhibition effect of miR-7, and inhibition of autophagy may enhance the antitumor effect of miR-7.

\section{Discussion}

$\mathrm{HCC}$ is one of the leading causes of cancer-associated mortality (11). Owing to the relatively low sensitivity to chemotherapy drugs, the prognosis of advanced HCC is poor (25). Understanding the underlying molecular mechanism will assist in the development of alternative effective strategies for HCC. Recently, the pharmaceutical value of miRNAs has drawn wide attention in cancer treatment $(26,27)$. A spectrum of miRNAs that are involved in cancer cell behavior have demonstrated therapeutic potential in HCC (16). The present study identified that miR-7, one of the downregulated miRNAs in HCC (21), functions as a tumor suppressor. Overexpression of miR-7 was able to significantly suppress the proliferation of HCC cells, and importantly, it was accompanied by increased autophagy. Furthermore, it was demonstrated that mTOR, a negative regulator of autophagy, is a direct target of miR-7. Pharmacological inhibition of autophagy using 3-MA enhanced the anti-proliferative activity of miR-7, indicating that the autophagy inhibitor may serve as the adjuvant drug to enhance the efficacy of miR-7 in the treatment of HCC.

Previous studies have depicted the crucial role of miRNAs in hepatocarcinogenesis $(14,28)$. miRNAs have been incorporated into a number of pathways to regulate cell proliferation, apoptosis, transdifferentiation and drug resistance. miR-7 has been demonstrated in a previous study to directly impede the normal function of several oncogenes in various types of cancer (16). A number of critical oncogenes, including epidermal growth factor receptor (EGFR), B-cell lymphoma-2, Akt and Krüppel-like factor-4 have been validated as the target of miR-7 (18,20,21), suggesting that miR-7 functions as the concurrent upstream regulator of multiple pathways that regulate cell survival. Fang et al (21) reported that $\mathrm{p} 70^{\mathrm{s} 6}$ kinase and mTOR are targeted by miR-7, which potentially provides the molecular basis for miR-7-based therapy for HCC. In the present study, it was confirmed that miR-7 was downregulated in $\mathrm{HCC}$, and consistent with the previous study (21), miR-7 overexpression exerted a significant proliferation inhibition action. Considering that mTOR is critical for the nutrient balance and multiple cellular events of cancer cells (10), the association between mTOR and miR-7 was then investigated. Consistent with the results of Fang et al (21), the present study demonstrated that mTOR was a target of miR-7.

In addition to examining the role of miR-7 in the proliferative activity of HCC cells, the present study focused on other critical processes. Autophagy is a lysosomal degradation process, through which diverse essential substances, including amino acids, lipids and nucleotides, are recycled $(3,5)$. It has been reported that autophagy at the physiological level is beneficial for cell survival, and uncontrolled autophagy may lead to cell death (4). Currently, studies on the role of autophagy in HCC are inconsistent. Luo et al (29) demonstrated that proteasome 26S subunit non-ATPase 10-induced autophagy promoted cell survival against starvation and chemotherapy. Tian et al (30) reported that autophagy is required for the prevention of hepatocarcinogenesis and the development of HCC. These studies suggested that autophagy may be a survival mechanism of
HCC cells. By contrast, other studies also demonstrated that autophagy inhibits tumorigenesis in HCC (31-33). Therefore, the exact role of autophagy in the development and progression of HCC is possibly context- and pathway-dependent. There are a few studies demonstrating the regulation of autophagy by miRNAs $(34,35)$. He et al $(36)$ demonstrated that miR-21 inhibits autophagy via the Akt/mTOR signaling pathway, which is important for sorafenib resistance of HCC cells. The present study identified that autophagy was significantly activated upon miR-7 overexpression. Similar to miR-21, a significant suppression of mTOR by miR-7 was observed, suggesting that the mTOR signaling pathway may also confer the induction of autophagy by miR-7. More importantly, inhibition of autophagy by 3-MA sensitized cells to miR-7-induced proliferation inhibition. These data indicated that autophagy may function as an adaptive survival mechanism to overcome the adverse effect of miR-7 on cancer cells. Notably, Tazawa et al (37) demonstrated that genetically engineered oncolytic adenovirus kills lung cancer cells through an autophagic cell death signaling pathway, which is mediated by the E2F transcription factorl/miR-7/EGFR axis. In this study, inhibition of autophagy partially rescued the decrease in cell viability caused by miR-7 overexpression, indicating an opposite role for autophagy in their system. This discrepancy may be a result of several differences in the stimulation type and the gene expression profile of the recipient cells. However, the mechanism for this phenomenon requires clarification in the future. Nonetheless, the results of the present study revealed that miR-7-induced autophagy is cytoprotective in the scenario of HCC.

In conclusion, the results of the present study revealed a novel mTOR-dependent mechanism by which miR-7 regulates autophagy. In addition, inhibition of autophagy enhanced the proliferation inhibition effect of miR-7. The present study suggested that modulating the levels of autophagy may be a feasible tool to enhance the therapeutic efficacy of miR-7 in the treatment of HCC.

\section{References}

1. Jemal A, Bray F, Center MM, Ferlay J, Ward E and Forman D: Global cancer statistics. CA Cancer J Clin 61: 69-90, 2011.

2. Balogh J, Victor D III, Asham EH, Burroughs SG, Boktour M, Saharia A, Li X, Ghobrial RM and Monsour HP Jr: Hepatocellular carcinoma: a review. J Hepatocell Carcinoma 3: 41-53, 2016.

3. Levine B and Kroemer G: Autophagy in the pathogenesis of disease. Cell 132: 27-42, 2008.

4. Mizushima N, Levine B, Cuervo AM and Klionsky DJ: Autophagy fights disease through cellular self-digestion. Nature 451: 1069-1075, 2008.

5. Puri $\mathrm{P}$ and Chandra A: Autophagy modulation as a potential therapeutic target for liver diseases. J Clin Exp Hepatol 4: 51-59, 2014.

6. Kumar A, Singh UK and Chaudhary A: Targeting autophagy to overcome drug resistance in cancer therapy. Future Med Chem 7: 1535-1542, 2015.

7. Du H, Yang W, Chen L, Shi M, Seewoo V, Wang J, Lin A, Liu Z and Qiu W: Role of autophagy in resistance to oxaliplatin in hepatocellular carcinoma cells. Oncol Rep 27: 143-150, 2012.

8. Jung $\mathrm{CH}$, Ro SH, Cao J, Otto NM and Kim DH: mTOR regulation of autophagy. FEBS Lett 584: 1287-1295, 2010.

9. Bupathi M, Kaseb A, Meric-Bernstam F and Naing A: Hepatocellular carcinoma: Where there is unmet need. Mol Oncol 9: 1501-1509, 2015.

10. Wang Z, Jin W, Jin H and Wang X: mTOR in viral hepatitis and hepatocellular carcinoma: Function and treatment. Biomed Res Int 2014: 735672, 2014. 
11. Inamura K: Diagnostic and therapeutic potential of MicroRNAs in lung cancer. Cancers (Basel) 9: pii: E49, 2017.

12. Cătană CS, Pichler M, Giannelli G, Mader RM and Berindan-Neagoe I: Non-coding RNAs, the Trojan horse in two-way communication between tumor and stroma in colorectal and hepatocellular carcinoma. Oncotarget 8: 29519-29534, 2017.

13. Faller M and Guo F: MicroRNA biogenesis: There's more than one way to skin a cat. Biochim Biophys Acta 1779: 663-667, 2008

14. Yao M, Wang L, Yao Y, Gu HB and Yao DF: Biomarker-based MicroRNA therapeutic strategies for hepatocellular carcinoma. J Clin Transl Hepatol 2: 253-258, 2014.

15. Horsham JL, Kalinowski FC, Epis MR, Ganda C, Brown RA and Leedman PJ: Clinical potential of microRNA-7 in cancer. J Clin Med 4: 1668-1687, 2015.

16. Kalinowski FC, Brown RA, Ganda C, Giles KM, Epis MR, Horsham J and Leedman PJ: microRNA-7: A tumor suppressor miRNA with therapeutic potential. Int J Biochem Cell Biol 54: 312-317, 2014.

17. Zhang X, Hu S, Zhang X, Wang L, Zhang X, Yan B, Zhao J, Yang A and Zhang R: MicroRNA-7 arrests cell cycle in G1 phase by directly targeting CCNE1 in human hepatocellular carcinoma cells. Biochem Biophys Res Commun 443: 1078-1084, 2014.

18. Xiong S, Zheng Y, Jiang P, Liu R, Liu X and Chu Y: MicroRNA-7 inhibits the growth of human non-small cell lung cancer A549 cells through targeting BCL-2. Int J Biol Sci 7: 805-814, 2011.

19. Luo J, Li H and Zhang C: MicroRNA-7 inhibits the malignant phenotypes of non-small cell lung cancer in vitro by targeting Pax6. Mol Med Rep 12: 5443-5448, 2015.

20. Chang YL, Zhou PJ, Wei L, Li W, Ji Z, Fang YX and Gao WQ MicroRNA-7 inhibits the stemness of prostate cancer stem-like cells and tumorigenesis by repressing KLF4/PI3K/Akt/p21 pathway. Oncotarget 6: 24017-24031, 2015.

21. Fang Y, Xue JL, Shen Q, Chen J and Tian L: MicroRNA-7 inhibits tumor growth and metastasis by targeting the phosphoinositide 3-kinase/Akt pathway in hepatocellular carcinoma. Hepatology 55: 1852-1862, 2012.

22. Livak KJ and Schmittgen TD: Analysis of relative gene expression data using real-time quantitative PCR and the 2(-Delta Delta C(T)) Method. Methods 25: 402-408, 2001.

23. Xie J, Chen M, Zhou J, Mo MS, Zhu LH, Liu YP, Gui QJ, Zhang L and Li GQ: miR-7 inhibits the invasion and metastasis of gastric cancer cells by suppressing epidermal growth factor receptor expression. Oncol Rep 31: 1715-1722, 2014.

24. Bitto A, Lerner CA, Nacarelli T, Crowe E, Torres C and Sell C: P62/SQSTM1 at the interface of aging, autophagy, and disease. Age (Dordr) 36: 9626, 2014.

25. Zhu AX: Systemic treatment of hepatocellular carcinoma: Dawn of a new era? Ann Surg Oncol 17: 1247-1256, 2010.
26. Gandellini P, Doldi V and Zaffaroni N: microRNAs as players and signals in the metastatic cascade: Implications for the development of novel anti-metastatic therapies. Semin Cancer Biol: Mar 23, 2017 (Epub ahead of Print).

27. Denaro N, Merlano MC, Russi EG and Lo Nigro C: Non coding RNAs in head and neck squamous cell carcinoma (HNSCC): A clinical perspective. Anticancer Res 34: 6887-6896, 2014.

28. Karagonlar ZF, Korhan P and Atabey N: Targeting c-Met in cancer by MicroRNAs: Potential therapeutic applications in hepatocellular carcinoma. Drug Dev Res 76: 357-367, 2015.

29. Luo T, Fu J, Xu A, Su B, Ren Y, Li N, Zhu J, Zhao X, Dai R, Cao J, et al: PSMD10/gankyrin induces autophagy to promote tumor progression through cytoplasmic interaction with ATG7 and nuclear transactivation of ATG7 expression. Autophagy 12: 1355-1371, 2016

30. Tian Y, Kuo CF, Sir D, Wang L, Govindarajan S, Petrovic LM and $\mathrm{Ou} \mathrm{JH}$ : Autophagy inhibits oxidative stress and tumor suppressors to exert its dual effect on hepatocarcinogenesis. Cell Death Differ 22: 1025-1034, 2015.

31. Huang GM, Jiang QH, Cai C, Qu M and Shen W: SCD1 negatively regulates autophagy-induced cell death in human hepatocellular carcinoma through inactivation of the AMPK signaling pathway. Cancer Lett 358: 180-190, 2015.

32. Wang Y, Wu J, Lin B, Li X, Zhang H, Ding H, Chen X, Lan L and Luo H: Galangin suppresses HepG2 cell proliferation by activating the TGF- $\beta$ receptor/Smad pathway. Toxicology 326: 9-17, 2014.

33. Lan SH, Wu SY, Zuchini R, Lin XZ, Su IJ, Tsai TF, Lin YJ, Wu CT and Liu HS: Autophagy suppresses tumorigenesis of hepatitis B virus-associated hepatocellular carcinoma through degradation of microRNA-224. Hepatology 59: 505-517, 2014.

34. Gozuacik D, Akkoc Y, Ozturk DG and Kocak M: Autophagy-Regulating microRNAs and cancer. Front Oncol 7: $65,2017$.

35. Liu L, Liao JZ, He XX and Li PY: The role of autophagy in hepatocellular carcinoma: Friend or foe. Oncotarget: Apr 18, 2017 (Epub ahead of print).

36. He C, Dong X, Zhai B, Jiang X, Dong D, Li B, Jiang H, Xu S and Sun X: MiR-21 mediates sorafenib resistance of hepatocellular carcinoma cells by inhibiting autophagy via the PTEN/Akt pathway. Oncotarget 6: 28867-28881, 2015.

37. Tazawa H, Yano S, Yoshida R, Yamasaki Y, Sasaki T, Hashimoto Y, Kuroda S, Ouchi M, Onishi T, Uno F, et al: Genetically engineered oncolytic adenovirus induces autophagic cell death through an E2F1-microRNA-7-epidermal growth factor receptor axis. Int J Cancer 131: 2939-2950, 2012. 